\title{
The National Early Warning Score 2 (NEWS2) in patients with hypercapnic respiratory failure
}

The National Early Warning Score (NEWS) was devised to standardise and improve the detection of, and response to, clinical deterioration in patients with acute illness. The original NEWS ${ }^{1}$ was released in 2012 and has been widely implemented across the NHS and in other healthcare settings globally. The NEWS has now been endorsed by NHS England and NHS Improvement as the single early warning score to be used by all acute hospitals and ambulance services, and it is anticipated that all NHS hospitals in England will be using the NEWS by 2019.

It was always anticipated that the NEWS would evolve, based on user experience and feedback, and in December 2017 the NEWS2 was released, ${ }^{2}$ containing important refinements. One of these changes related to the safer use of oxygen in patients with chronic hypercapnic respiratory failure (HCRF), most commonly seen in some patients with acute exacerbations of chronic obstructive pulmonary disease (AECOPD). For such patients, the recommended oxygen saturation range is $88-92 \%$ and these patients are at risk of rapidly worsening hypercapnia and death if too much oxygen is delivered. ${ }^{3}$ Concern had been expressed that the original NEWS automatically generated a high score for patients with HCRF due to their hypoxia, prompting use of supplemental oxygen (even though their $\mathrm{SpO}_{2}$ may be in the desired range), leading to frequent alerts and ultimately decisions to override the score or apply ad hoc adjustments. There was concern that the chronically high score in patients with HCRF might encourage the inappropriate use of additional supplemental oxygen in an attempt to reduce the NEWS score by increasing oxygen saturations above the recommended range for these patients. 4,5

After much discussion and consultation with the British Thoracic Society and others, a new scoring $\mathrm{SpO}_{2}$ scale (scale 2) was added to the NEWS2 chart that was specifically designed for use only in patients with documented HCRF, explicitly noting that most acutely ill patients with AECOPD will not present with $\mathrm{HCRF}^{2}$ Supporting this change, audits of hospital care had shown that administration of high-flow oxygen, rather than titrated oxygen, to patients with AECOPD increases mortality, hospital length of stay, requirement for ventilation and admission to higher-dependency care. In contrast, the use of appropriately titrated oxygen resulted in less acidosis and reduced mortality. ${ }^{6-10}$

In the previous issue of Clinical Medicine, Hodgson and colleagues question the validity of this change. ${ }^{11}$ In 2013 , several of the same authors published comments consistent with the rationale for the NEWS2 when they wrote "We have shown that the current NEWS system leads to a significant number of patients with AECOPD alerting when they have nationally recommended target oxygen saturations. A simple adjustment of the alerting threshold in this cohort could improve the system. ${ }^{12}$

The conclusions in the recent paper ${ }^{11}$ suggest that applying the NEWS2 $\mathrm{SpO}_{2}$ scale 2 to patients admitted to hospital with AECOPD reduced the efficiency of the NEWS2 in detecting acutely ill patients with AECOPD compared with the original NEWS. At face value, the data look compelling. However, the analysis and conclusions are unreliable because the authors have misused the NEWS2 scoring system in their analysis. They have inappropriately applied the new $\mathrm{SpO}_{2}$ scale 2 to all patients with AECOPD at first assessment, rather than just those with documented HCRF for whom the scale is specifically designed. The NEWS2 report ${ }^{2}$ clearly states 'For patients confirmed to have hypercapnic respiratory failure on blood gas analysis on either a prior or their current hospital admission, and requiring supplemental oxygen, we recommend (i) a prescribed oxygen saturation target range of $88-92 \%$, and (ii) that the dedicated $\mathrm{SpO}_{2}$ scoring scale (scale 2) on the NEWS2 chart should be used to record and score the oxygen saturation for the NEWS.' and 'The decision to use $\mathrm{SpO}_{2}$ scale 2 should be made by a competent clinical decision maker and should be recorded in the patient's clinical notes,' and, critically, 'In all other circumstances,

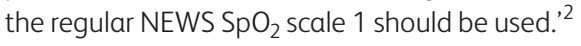

The NEWS2 $\mathrm{SpO}_{2}$ scale 2 should not be used for the initial assessment of patients with AECOPD, as was done by Hodgson et al. ${ }^{11}$ The $\mathrm{SpO}_{2}$ scale 1 should have been used; this is identical to the original NEWS, which obviously would have generated identical specificities and sensitivities for the NEWS and NEWS2. Patients should only be converted to $\mathrm{SpO}_{2}$ scale 2 if they have documented HCRF, and after review and a decision by a competent clinical decision-maker. ${ }^{2}$ What this study ${ }^{11}$ actually shows is that if the NEWS2 scoring system is not used properly and as clearly described, it may not work as well. There is clearly an important need for further education about the use of the NEWS2 $\mathrm{SpO}_{2}$ scoring scales in patients with AECOPD. For the avoidance of doubt, the NEWS2 $\mathrm{SpO}_{2}$ scale 1 should be used in all patients at first assessment, unless there is clear documented evidence of HCRF, validated by a competent clinical decision-maker - then, and only then, the $\mathrm{SpO}_{2}$ scale 2 can be used.

BRYAN WILLIAMS

Chair of medicine, University College London, London, UK Chairman of the NEWS and NEWS2 Development

Groups for the RCP

\section{References}

1 Royal College of Physicians. National Early Warning Score (NEWS): Standardising the assessment of acute-illness severity in the NHS. Report of a working party. London: RCP, 2012. 
2 Royal College of Physicians. National Early Warning Score (NEWS) 2: Standardising the assessment of acute-illness severity in the NHS. Updated report of a working party. London: RCP, 2017.

3 O'Driscoll BR, Howard LS, Earis ] et al. British Thoracic Society Emergency Oxygen Guideline Group. BTS guideline for oxygen use in adults in healthcare and emergency settings. Thorax 2017;72(Suppl 1):ii1-90.

4 Kane B, Decalmer S, Murphy P, Turkington P, O'Driscoll BR. S29 The proposed National Early Warning System (NEWS) could be hazardous for patients who are at risk of hypercapnic respiratory failure. Thorax 2012;67(Suppl 2):A16-A17.

5 O'Driscoll BR, Grant K, Green D et al. The National Early Warning Score gives misleading scores for oxygen saturation in patients at risk of hypercapnia. Clin Med 2014;14:695-6.

6 Denniston AKO, O'Brien C, Stableforth D. The use of oxygen in acute exacerbations of chronic obstructive pulmonary disease: a prospective audit of pre-hospital and hospital emergency management. Clin Med 2002;2:449-51.

7 Joosten SA, Koh MS, Bu X, Smallwood D, Irving LB. The effects of oxygen therapy in patients presenting to an emergency depart- ment with exacerbation of chronic obstructive pulmonary disease. Med J Aust 2007;186:235-8.

8 Plant PK, Owen JL, Elliott MW. One year period prevalence study of respiratory acidosis in acute exacerbations of COPD: implications for the provision of non-invasive ventilation and oxygen administration. Thorax 2000;55:550-4.

9 Wijesinghe M, Perrin K, Healy B et al. Pre-hospital oxygen therapy in acute exacerbations of chronic obstructive pulmonary disease. Intern Med J 2011;41:618-22.

10 Austin MA, Wills KE, Blizzard L, Walters EH, Wood-Baker R. Effect of high flow oxygen on mortality in chronic obstructive pulmonary disease patients in prehospital setting: randomised controlled trial. BM] 2010;341:c5462.

11 Hodgson LE, Congleton J, Venn R, Forni LG, Roderick PJ. NEWS 2 - too little evidence to implement? Clin Med 2018;18: 371-3.

12 Hodgson L, Bax S, Montefort M et al. S68 The National Early Warning Score (NEWS) \& iatrogenic harm - could the NEWS for COPD patients be improved? Thorax 2013;68: A37. 


\section{9:2 Royal College Q. of Physicians}

\section{Under the skin \\ Illustrating the human body \\ 1 February - 15 March 2019}

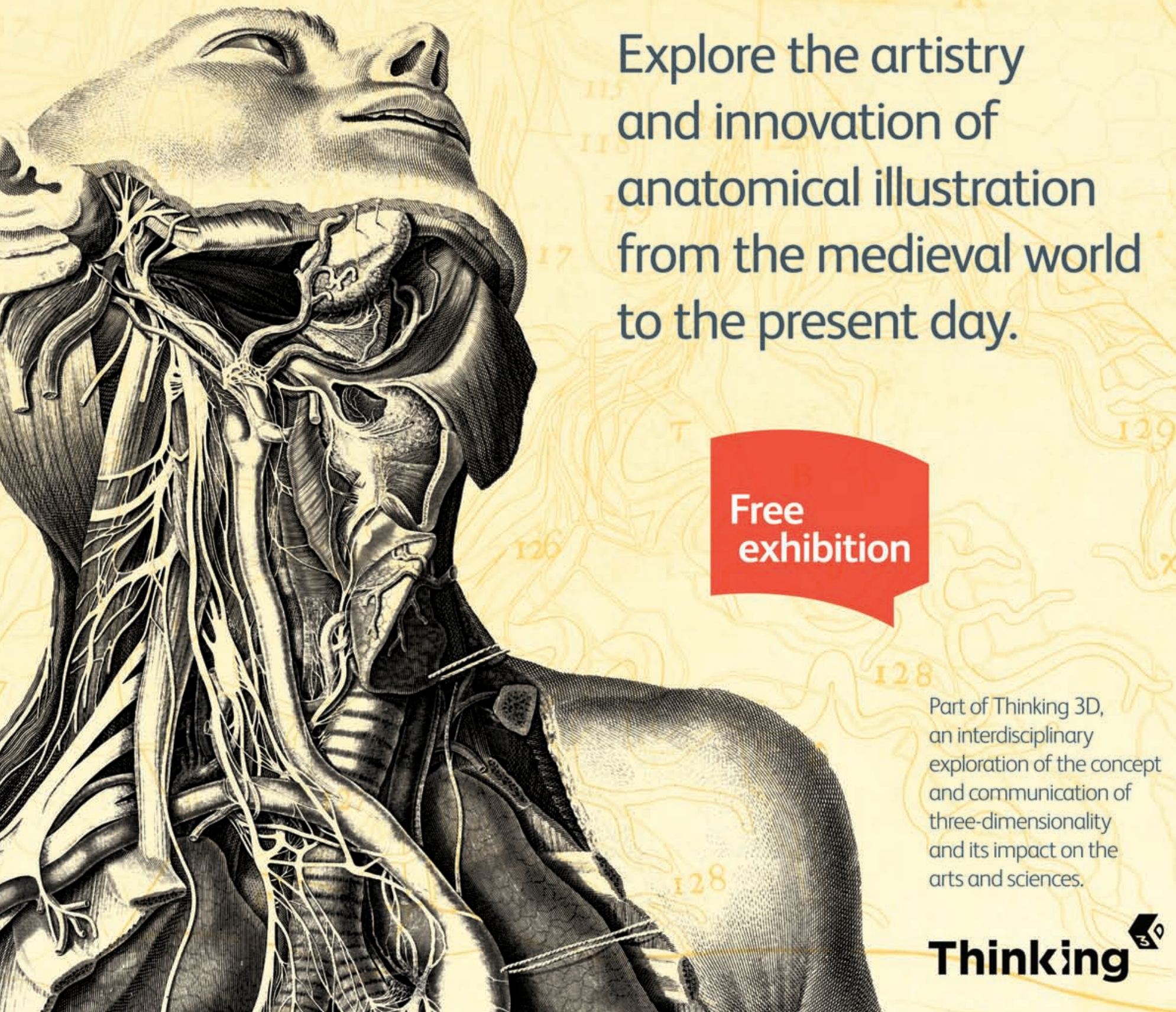

